\title{
Identification of Two Variant Short Chain Acyl-Coenzyme A Dehydrogenase Alleles, Each Containing a Different Point Mutation in a Patient with Short Chain Acyl-Coenzyme A Dehydrogenase Deficiency
}

\author{
Etsuo Naito, Yasuhiro Indo, and Kay Tanaka \\ Yale University School of Medicine, Department of Human Genetics, New Haven, Connecticut 06510
}

\begin{abstract}
Two distinct mutant alleles of the precursor (p) short chain acyl-CoA dehydrogenase (SCAD) gene were identified in a SCAD-deficient patient (YH2065) using the polymerase chain reaction to amplify cDNA synthesized from total RNA from her fibroblasts. Cells from this patient had previously been shown to synthesize a labile variant SCAD in contrast to the normal stability of variant SCADs in two other SCAD-deficient cell lines (Naito, E., Y. Indo, and K. Tanaka. 1989. J. Clin. Invest. 84:1671-1674). In the present study, both mutant alleles of YH2065 were found to contain a $C \rightarrow T$ transition, one at position 136 and the other at position 319 of the coding region of pSCAD cDNA. Clones of cDNA amplified from this region showed only one of the $\mathrm{C} \rightarrow \mathrm{T}$ transitions, indicating that each mutation was derived from different pSCAD alleles. Each of these mutations altered a known restriction endonuclease site, and restriction analysis of additional cDNA clones from amplified mutant cDNA and Southern blotting of mutant genomic DNA confirmed the presence of two unique mutant alleles in YH2065, indicating YH2065 is a compound heterozygote. These $\mathrm{C} \rightarrow \mathrm{T}$ transitions result in the substitution of Arg-22 and Arg-83 of the mature SCAD with Trp and Cys, respectively. (J. Clin. Invest. 1990. 85:1575-1582.) short chain acyl-CoA dehydrogenase $\bullet$ variant alleles $\bullet$ point mutation $\bullet$ sequence analysis $\bullet$ altered restriction sites
\end{abstract}

\section{Introduction}

Short chain acyl-CoA dehydrogenase (SCAD) ${ }^{1}$ deficiency is an inborn error of fatty acid metabolism that has recently been reported in three infants $(1,2)$. The main clinical symptoms in two of them were an early episode of lethargy and metabolic acidosis, whereas in the other, progressive skeletal muscle weakness, developmental delay, and evidence of fatty infiltration of muscle and liver were the prominent features (2). In

Address reprint requests to Dr. K. Tanaka, Yale University School of Medicine, Department of Human Genetics, 333 Cedar Street, P.O. Box 3333, New Haven, CT 06510.

Received for publication 20 October 1989 and in revised form 10 January 1990.

1. Abbreviations used in this paper: IVD, isovaleryl-CoA dehydrogenase; MCAD, medium chain acyl-CoA dehydrogenase; $p$, precursor; PCR, polymerase chain reaction; SCAD, short chain acyl-CoA dehydrogenase.

J. Clin. Invest.

(C) The American Society for Clinical Investigation, Inc.

0021-9738/90/05/1575/08 \$2.00

Volume 85, May 1990, 1575-1582 our previous studies $(3,4)$, we have shown that the cells from all three patients synthesized a variant SCAD protein of normal size. This suggested that the defect of SCAD in these patients is caused by a point mutation in the SCAD gene (3). Furthermore, using immunoblot analysis and pulse-chase experiment, we showed that variant SCAD synthesized by the cells from one of the patients, reported by Amendt et al. as L.N. (1) and coded by us as YH2065 (3, 4), was labile, whereas the stability of the variant SCAD protein in the cells from two other patients was comparable to normal cells, indicating heterogeneity of the point mutations (4).

The biochemical function of SCAD is to dehydrogenate butyryl-CoA, initiating the $\beta$-oxidation cycle for this substrate at the last stage of fatty acid oxidation $(5,6)$. It is a member of the acyl-CoA dehydrogenase family (7). Other enzymes in this gene family are long chain acyl-CoA dehydrogenase and medium chain acyl-CoA dehydrogenase (MCAD), isovalerylCoA dehydrogenase (IVD), and 2-methyl-branched chain acyl-CoA dehydrogenase (7). Like four other members of the gene family, SCAD is a tetrameric mitochondrial flavoenzyme $(5,6)$. Its $41-\mathrm{kD}$ subunit is encoded in the nucleus and synthesized in the cytosol as a precursor (p) that contains a $3-\mathrm{kD}$ extra peptide (leader peptide) at the $\mathrm{NH}_{2}$-terminus of the mature enzyme that is required for the transport of pSCAD to the mitochondria. The subunit precursor is then imported into mitochondria, proteolytically processed into the mature subunit, and assembled into the tetrameric form (8).

We have recently reported the cloning and sequencing of a cDNA encoding human pSCAD (3). The coding region of human pSCAD cDNA is 1,236 -bp long, and encodes the $412-$ amino acid pSCAD. The first 72 nucleotides at the 5 '-end encode the 24-amino acid leader peptide that is contiguous to the $\mathrm{NH}_{2}$-terminus of the 388-amino acid mature SCAD (3). With these sequence data in hand, we sought to characterize the nature of mutation(s) responsible for SCAD-deficiency at the gene level using the polymerase chain reaction (PCR). This technique provides a convenient procedure to greatly enrich the amount of a specific DNA sequence, even when present in minute amounts, thereby fascilitating the analysis of DNA sequence $(9,10)$. In this paper, we report the identification of two distinct variant SCAD alleles in YH2065 cells, each with a point mutation in an arginine codon at different positions.

\section{Methods}

Radioisotopes and chemicals. $\left[{ }^{35} \mathrm{~S}\right] \mathrm{dATP}$ and $\left[{ }^{32} \mathrm{P}\right] \mathrm{dCTP}$ were purchased from Amersham Corp. (Arlington Heights, IL). Most reagents and enzymes for molecular cloning were procured from Promega Biotec (Madison, WI). Taq DNA polymerase was from Perkin-Elmer Cetus (Norwalk, CT). Random hexamer primers pd(N) 6 were from Pharmacia LKB Biotechnology (Piscataway, NJ) and oligo(dT) was from Bethesda Research Laboratories (Gaithersburg, MD). The ran- 
dom-primed labeling kit was from Boehringer Mannheim Biochemicals (Indianapolis, IN). Guanidium isothiocyanate was obtained from Eastman Kodak Co. (Rochester, NY). Cell culture materials were from Gibco Laboratories (Grand Island, NY)

Source of cells and culture method. The sources of the three SCAD-deficient cells have been previously reported $(3,4)$. The normal cell lines were obtained from the NIGMS Human Genetic Mutant Cell Repository (Camden, NJ). Fibroblasts were grown in monolayer culture in Eagle's minimum essential medium supplemented with $10 \%$ fetal calf serum and kanamycin (Gibco Laboratories) at $37^{\circ} \mathrm{C}$ in $5 \%$ $\mathrm{CO}_{2}$ atmosphere. The cells were grown to confluence and harvested by trypsinization 3-6 d after the last medium change.

RNA isolation and cDNA synthesis. Total cellular RNA was isolated from SCAD-deficient (YH2065) and normal (GM05756) cell lines, and normal human liver using guanidium isothiocyanate as described previously (3). Human liver poly(A) $)^{+}$RNA was separated from total RNA by oligo(dT)-cellulose chromatography. First-strand cDNAs were synthesized from total RNA or poly(A) ${ }^{+}$RNA using Moloney murine leukemia virus reverse transcriptase and a cDNA synthesis kit (Bethesda Research Laboratories) as follows: $10 \mu \mathrm{g}$ of total RNA or $5 \mu \mathrm{g}$ of poly(A) ${ }^{+}$RNA was mixed on ice with either $5 \mu \mathrm{g}$ of random hexamer primers pd(N) ${ }_{6}$ when the $5^{\prime}$ - and middle fragments were to be amplified subsequently or $2.5 \mu \mathrm{g}$ of oligo(dT) for subsequent amplification of the 3 '-fragment. Transcription was then carried out at $37^{\circ} \mathrm{C}$ for $60 \mathrm{~min}$ in $50 \mu \mathrm{l}$ of reaction mixture containing $50 \mathrm{mM}$ Tris- $\mathrm{HCl}$ (pH 8.3), $75 \mathrm{mM} \mathrm{KCl}, 3 \mathrm{mM} \mathrm{MgCl}_{2}, 10 \mathrm{mM}$ DTT, $0.5 \mathrm{mM}$ dNTPs (dATP, dCTP, dGTP, and dTTP) and $2.5 \mu$ of the above reverse transcriptase.

Synthesis of PCR primers, and amplification of the human SCAD $m R N A$ and genomic DNA. Three pairs of 30-mer primers were selected as described under Results and synthesized using a DNA synthesizer (model 380A, Applied Biosystems, Inc., Foster City, CA). In each pair, the upstream primer corresponded to the RNA sense strand sequence, and the downstream primer corresponded to the antisense strand sequence of normal human SCAD mRNA (3). The primers were purified using an oligonucleotide purification cartridge (Applied Biosystems, Inc.) (11). PCR amplification was carried out using an appropriate aliquot (typically, 8-10 $\mu$ l) of the first-strand synthesis mixture as template and $1 \mu \mathrm{M}$ of each of a pair of the PCR primers. The reaction mixture contained $10 \mathrm{mM}$ Tris- $\mathrm{HCl}, \mathrm{pH} 8.3,3.5 \mathrm{mM} \mathrm{MgCl}$, $50 \mathrm{mM} \mathrm{KCl}, 0.01 \%$ gelatin, and $200 \mu \mathrm{M}$ each of dATP, dCTP, dGTP, and dTTP in $100 \mu \mathrm{l}$. The reaction mixture was boiled for $2 \mathrm{~min}$, cooled to room temperature, $0.5 \mu \mathrm{l}$ of Taq DNA polymerase was added, and the reaction mixture was covered with $100 \mu \mathrm{l}$ mineral oil (Brand-Nu Laboratories, Inc., Meriden, CT). 30 cycles of PCR were performed in a DNA thermal cycler (Perkin-Elmer Cetus) using the following profile: $1 \mathrm{~min}$ denaturation at $94^{\circ} \mathrm{C}$, followed by $2 \mathrm{~min}$ annealing at $50^{\circ} \mathrm{C}$, and 3 min extension at $72^{\circ} \mathrm{C}$.

For PCR amplification of genomic DNA sequences corresponding to the middle 118-bp section of cDNA, high molecular weight genomic DNA was isolated from three SCAD-deficient and three normal cell lines using guanidium isothiocyanate as described previously (3). Genomic DNA $(1 \mu \mathrm{g})$ was annealed to $1 \mu \mathrm{M}$ each of the pair of primers for the middle 118-bp fragment. After boiling the samples for $2 \mathrm{~min}, 1 \mu \mathrm{l}$ of Taq DNA polymerase was added, and 30 cycles of the reactions were carried out as described above.

Subcloning of PCR amplified products. The PCR-amplified product derived from total RNA was first digested with Hinc II, Taq I, or Bam HI, and purified by electrophoresis using a $1.5 \%$ low-melting agarose gel (SeaPlaque; FMC BioProducts, Rockland, ME). Fragments with the expected size were excised, extracted with phenol/chloroform and precipitated with ethanol. They were then subcloned into pBluescript vector (Stratagene, La Jolla, CA), which had been digested with Hinc II, Acc I or Bam HI. The 118-bp PCR amplified product from genomic DNA was digested with both Taq I and Hinc II, and then subcloned into bluescript vector which had been digested with Acc and Sma I. The newly constructed plasmid was amplified by transforming Escherichia coli strain XL1-Blue (Stratagene).
DNA sequencing. DNA sequencing was performed directly using the recombinant pBluescript plasmid with its CDNA insert by the dideoxy-sequencing method (12) using an appropriate oligonucleotide (T7 or T3; Stratagene), $\left[{ }^{35}\right.$ S $]$ dATP and the Sequenase kit (United States Biochemical Corporation, Cleveland, $\mathrm{OH})$.

Digestion of the PCR products by restriction enzyme. After PCR, the reaction mixture was extracted with chloroform to remove the mineral oil. Two 20- $\mu$ l aliquots were digested with either Hpa II or Pvu II, and the resulting DNA fragments were resolved by electrophoresis on a 4\% agarose gel (NuSieve; FMC BioProducts) containing ethidium bromide. The separated DNA fragments were visualized with ultraviolet light.

Southern blot analysis of genomic DNA. $10 \mu \mathrm{g}$ each of genomic DNA isolated from three SCAD-deficient and three normal cell lines were digested with Hpa II or Pvu II. The resulting fragments were separated on a $1.0 \%$ agarose gel (Bio-Rad Laboratories, Richmond, $\mathrm{CA}$ ) and transferred onto a sheet of Hybond-N membrane (Amersham Corp.) according to the manufacturer's protocol. The membrane was then hybridized using a 489-bp fragment (nucleotide position 23-511) of human SCAD cDNA as a probe. Final washes were performed at $65^{\circ} \mathrm{C}$ in $0.2 \times$ SSPE/0.1\% SDS. Blots were exposed to Kodak X-AR film for autoradiography.

Prediction of the secondary structure of variant SCAD using a computer. A computer-assisted prediction of the secondary structure perturbations in variant SCAD caused by an amino acid substitution was performed by the method of Chou and Fasman (13) using the University of Wisconsin Genetics Computer Group software developed by Wolf et al. (14). Hydrophilicity/hydrophobicity moments were also calculated and overlaid on the secondary structure plots (15).

\section{Results}

\section{Selection of primers for PCR}

We planned to amplify the entire SCAD cDNA-coding region and some untranslated flanking region in three sections, using three sets of 30 oligonucleotide primers that were selected and synthesized according to the normal human SCAD cDNA sequence (3) as illustrated in Fig. 1. The segment between each pair of primers overlapped the neighboring one(s), such that the entire coding region plus some in the flanking regions of the SCAD mRNA sequence could be determined. In order to facilitate the isolation of the PCR products, primers were selected in such a way that each contained an appropriate restriction site for subcloning. Natural restriction sites are present in the primer pair for the middle fragment and in the downstream primer for the 5 -fragment. 2,1 , and 1 nucleotide mismatches, respectively, were introduced into the primers for the 3 -fragment and the upstream primer for the 5 -fragment to create an appropriate restriction site. The three restriction enzymes were selected because these enzymes produced no restriction fragment length polymorphism in two normal and three SCAD-deficient cell lines (3). The sizes of the expected amplification products are 399,638 , and 499 bp for the 5'-, middle, and 3 '-fragments, respectively.

\section{Amplification of the human SCAD mRNA using PCR}

As shown in Fig. $2 A$, the size of the major band in each of the 5'-, middle and 3'-fragments amplified from cloned human SCAD cDNA (HS-1) exactly correspond to the predicted sizes shown in Fig. 1. In some of the amplification products from total RNA from a normal and YH2065 cell, a single major band was detected. In others, numerous bands were detected, but the size of the major band in each was identical to the corresponding band amplified from the cloned SCAD cDNA. 


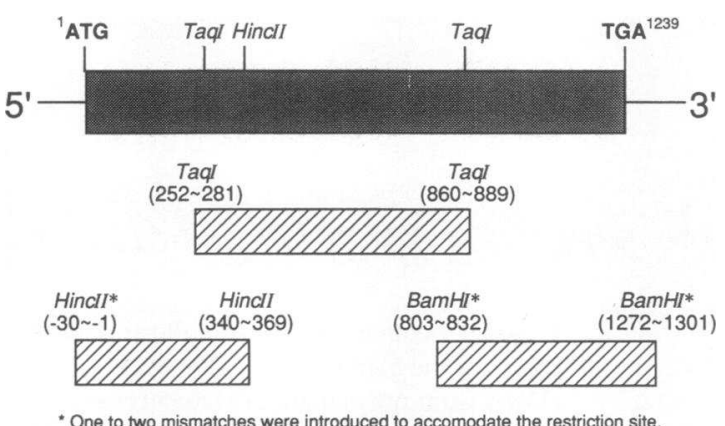

Figure 1. Strategy for PCR amplification of pSCAD cDNA, subcloning, and sequencing of the amplified fragments. The sections shown with a shaded rectangle and lines on both sides indicate the coding and noncoding region of human precursor SCAD, respectively. The base number for the A of the ATG initiation codon and the terminal A of the TGA stop codon are indicated as 1 and 1,239 , respectively. The position of restriction sites, which exist in the coding region of pSCAD and used for subcloning of the amplified fragments, is indicated on the top rectangle. The six PCR primers were used to amplify three sections of pSCAD, shown each with hatched rectangles, from first-strand cDNA synthesized from total RNA. Each primer was selected so that each pair contained an appropriate cloning site (Hinc II, Taq I, or Bam HI). When an appropriate endogenous restriction site was not present, one or two mismatches were introduced to accommodate the restriction site. These are marked with an asterisk. The location indicated in parentheses with base number is according to the published pSCAD cDNA sequence (3). The expected sizes of the PCR-amplified copies are 399, 638, and $499 \mathrm{bp}$, respectively.

Minor bands seen were presumably produced by nonspecific priming of other RNAs.

To definitively identify the bona-fide SCAD fragment in the PCR-amplification products, the same gel was further analyzed by Southern blot hybridization using the cloned human cDNA (HS-1) as a probe. In each lane, only the major band anticipated to correspond to SCAD sequence specifically hybridized (Fig. $2 \mathrm{~B}$ ), positively identifying it as an authentic copy of the intended segment of human SCAD cDNA.

Identification of point mutations in YH2065 cell line. In the determination of the nucleotide sequences of the amplified cDNA fragments, we carried out the entire process including the RNA isolation, reverse transcription, and PCR amplification independently two to three times for each of the three fragments of mRNAs from YH2065, normal fibroblasts, and human liver, and each product was subcloned in order to exclude transcription errors in cDNA synthesis or in amplification by the PCR. Multiple clones of each product from each round of amplification were sequenced. In the systematic nomenclature for identifying the position of the nucleotide and amino acid substitutions in the following description, the positions in the sequence of the precursor SCAD is used unless otherwise noted.

In all, five different nucleotide substitutions, not matching the previously reported normal human SCAD cDNA sequence (3), were consistently detected in YH2065 in each round of amplification. Three of them were in the 5 -fragment involving the coding sequence, whereas two others were in the noncoding region in the 3 '-fragment. One of the three substitutions found in the $5^{\prime}$-fragment was a $\mathrm{C} \rightarrow \mathrm{T}$ transition at

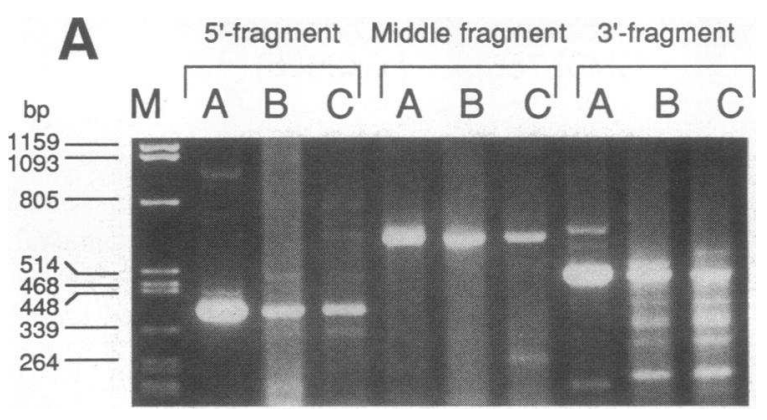

B

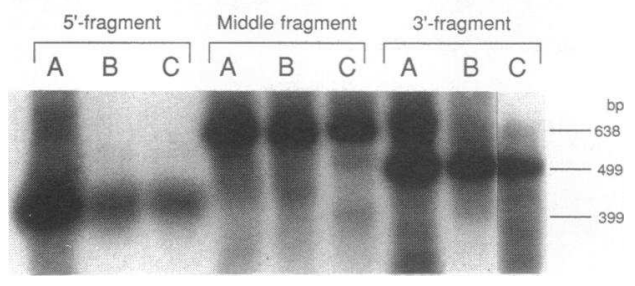

Figure 2. PCR amplification of the first-strand cDNA synthesized from total RNA from normal and SCAD-deficient fibroblasts. The coding region of the human SCAD mRNA was amplified in three sections from cDNA generated by reverse transcription of the total cellular RNA of the fibroblasts. $20 \mu \mathrm{l}$ of the reaction mixture was analyzed on a $1.5 \%$ agarose gel containing ethidium bromide. Lane $M$, the molecular weight markers with Pst I-digested $\lambda D N A$; lane $A$ in each section, the PCR-amplified fragments generated from SCAD cDNA (HS-1) (3); lane $B$ in each section, the PCR-amplified fragments derived from a normal cell line; and lane $C$ in each section, the PCR-amplified fragments derived from YH2065 cell line. $(A)$ Analysis on ethidium bromide-stained gel. $(B)$ Southern blot analysis of the PCR products. The ethidium bromide-stained gel shown in Fig. 2 was denatured, transferred to a Hybond-N membrane, and hybridized with the insert of human pSCAD cDNA clone (HS-1) (3). Fragment sizes were calculated based on the size of Pst I-digested ADNA fragments, which were electrophoresed in the same gel.

position 136 (counted taking the $A$ in the initiation codon as 1 ) in a CGG codon. This point mutation alters the 46th amino acid residue in the pSCAD, changing Arg- 22 in the mature SCAD, to $\operatorname{Trp}$ (Fig. $3 A$ ). This $\mathrm{C} \rightarrow \mathrm{T}$ transition was detected in three of the six clones from the first round of amplification. Two other substitutions found in the 5'-fragment from YH2065 both involved the CGT codon encoding the 107th residue of pSCAD that is Arg-83 of the mature SCAD. One was a $\mathrm{C} \rightarrow \mathrm{T}$ transition at position 319 , that was found in three remaining clones from the first round amplification. This transition caused a substitution of Arg-83 with Cys (Fig. 3 B). These two $C \rightarrow T$ transitions were consistently found in the products from the later amplification experiments with YH2065 mRNA, but not from those of normal mRNA. In the independently repeated experiments, the $C \rightarrow T$ 136-transition was found in 12 of the 24 clones, and in the remaining 12 clones, C $\rightarrow$ T-319 transition was found. We designated these mutations R46W and R107C, respectively. The other substitution involving the CGT codon for Arg-83 (mature SCAD) was a $\mathrm{T} \rightarrow \mathrm{C}$ transition at nucleotide 321 , producing CGC. However, this appears to be a normal polymorphism since both CGT and CGC encode Arg, and both of them are repeatedly found in the amplified products from normal mRNA. 


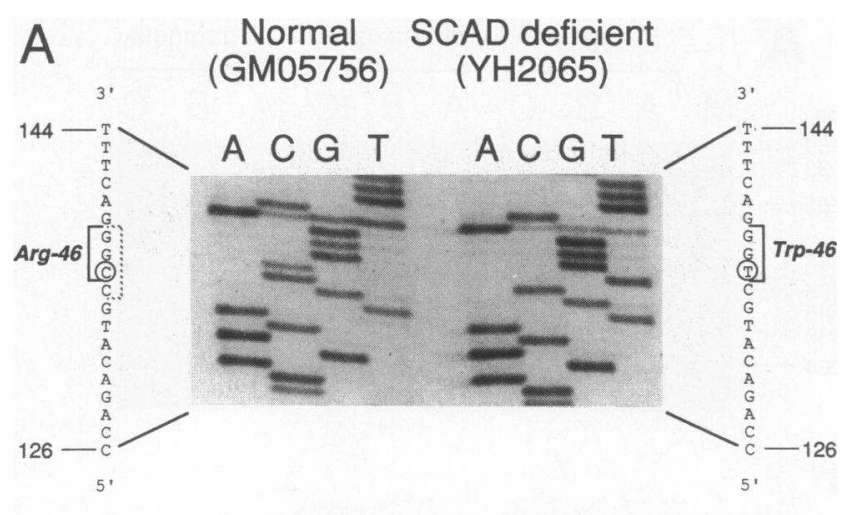

The dotted line indicates a HpaII site.

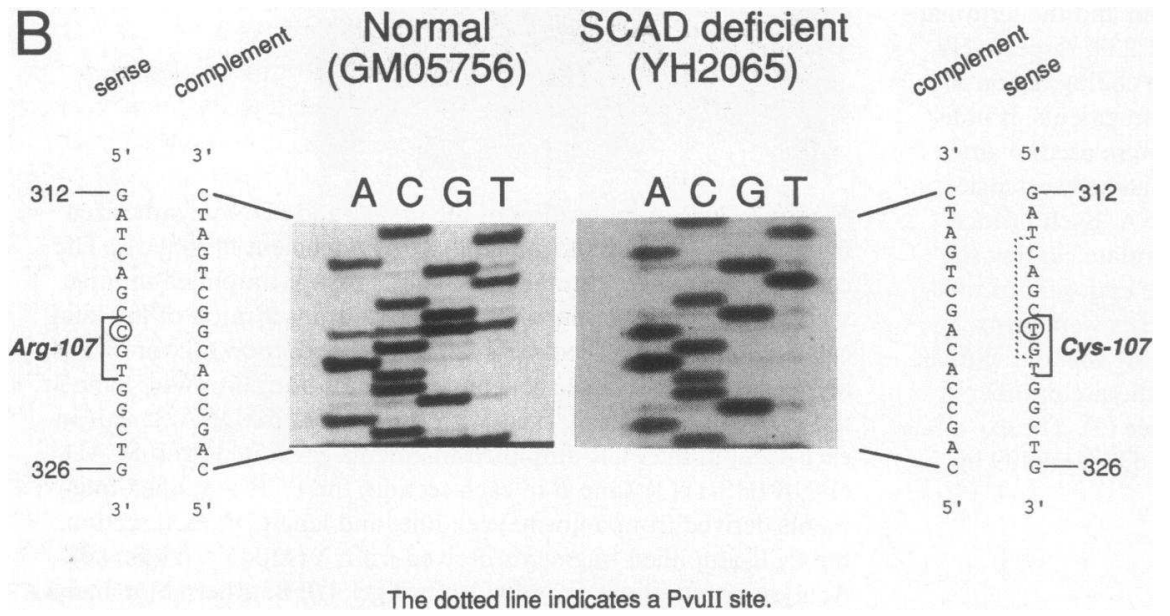

Figure 3. Sequence analysis of the amplified copies of the 5'-fragments of the SCAD cDNA from normal and YH2065 cells. In the first round of amplification of the 5'-fragment of YH2065 mRNA, the amplified copies were subcloned. Six clones were sequenced. The nucleotide residues involved in the mutations are circled. In $A$, the sequence from positions 126-144 found in three of the clones are shown on the right side with the corresponding part of the amplified copy from normal cells at the left. $\mathrm{C}-136$ in the normal cDNA is substituted with T in YH2065 cDNA. An Hpa II site $(C C G G)$ in normal SCAD sequence, indicated by a broken line, is abolished by this transition $(C T G G)$ at nucleotide 136 . In $B$, the sequence of the reverse strand from positions $312-326$ of pSCAD cDNA from normal cells and that found in three other clones of YH2065 are shown on the left and right sides, respectively. C-319 in the normal cDNA is substituted with $\mathrm{T}$ in $\mathrm{YH} 2065$ cDNA. This nucleotide substitution created a restriction site for Pvu II (CAGCTG) as shown by a broken line.
These results indicate that there are two variant alleles, one carrying R46W and the other carrying R $107 \mathrm{C}$.

The two substitutions found in the 3 '-noncoding region were a $\mathrm{G} \rightarrow$ A transition at position 1,246 and a $\mathrm{G} \rightarrow \mathrm{C}$ transversion at position 1,260 . In addition to the five substitutions that were constantly found, we detected another nucleotide substitution, an $\mathrm{A} \rightarrow \mathrm{T}$ transversion, at position 346 in the coding region of a single clone. However, this substitution was not detected in the fragments from the later rounds of amplification. Therefore, it was assumed to be a transcription artifact.

Digestion of the PCR products with restriction enzymes. In order to further show that the R46W and R107C substitutions are intrinsic to the variant alleles, we took advantage of the changes of restriction sites caused by these transitions. As shown in Fig. $3 A$, the tetranucleotide, CCGG, including C-136, constitutes a Hpa II site that would be abolished by the $\mathrm{C} \rightarrow \mathrm{T}-136$ transition. The anticipated results of this digestion is illustrated in Fig. $4 \mathrm{~B}$. There are three $\mathrm{Hpa}$ II sites in the 5 '-fragment from normal SCAD mRNA. Thus, digestion of the $5^{\prime}$-fragments from normal cells with $\mathrm{Hpa}$ II produces four fragments $(200,114,51$, and $34 \mathrm{bp}$, respectively). In contrast, digestion of the amplified copies of the 5 '-fragments from YH2065 is expected to produce five fragments, four normalsized fragments, plus an additional longer fragment $(314 \mathrm{bp})$ from allele-R46W. The results of the electrophoretic analysis of the Hpa II digestion products are shown in Fig. $4 \mathrm{~A}$. In YH2065, a strong 314-bp band was detected in addition to four shorter normal fragments as anticipated (lane 6), whereas this band was not detected in normal cells. Furthermore, in YH2065, the intensity of the 114- and 200-bp bands were considerably weaker than in the normal cell line, indicating that the $\mathrm{C} \rightarrow \mathrm{T}-136$ transition is indeed intrinsic to only one of the two variant alleles in YH2065.

In allele-R $107 \mathrm{C}$, a Pvu II site is created due to the $\mathrm{C} \rightarrow$ T-319 transition whereas no Pvu II site is present in normal human SCAD cDNA. Thus, when the 5'-fragments from normal and R46W alleles are treated with Pvu II, they remain undigested. In contrast, digestion of the 5 '-fragment from allele-R 107C with Pvu II is expected to produce two fragments, one with the size of $347 \mathrm{bp}$ and the other with the size of $52 \mathrm{bp}$. When total amplified 5'-fragment copies from YH2065 were directly digested with Pvu II, three fragments (399, 347, and 52 bp) were indeed detected, whereas the size of the normal 5'fragment ( $399 \mathrm{bp}$ ) did not change after treatment with Pvu II (Fig. $4 \mathrm{~A}$, lanes 7 and 8 ). The undigested 399-bp piece in the Pvu II digest of YH2065 5'-fragment is presumably due to allele-R46W.

Identification of the $C \rightarrow T-319$ transition in genomic $D N A$. To identify the presence of the $\mathrm{C} \rightarrow \mathrm{T}-319$ transition in genomic DNA, a short segment of genomic DNA was amplified by PCR using the upstream primer for the middle fragment and the down-stream primer for the 5 -fragment. The size of the product of this amplification is expected to be 118 bp encompassing positions 252-369. 


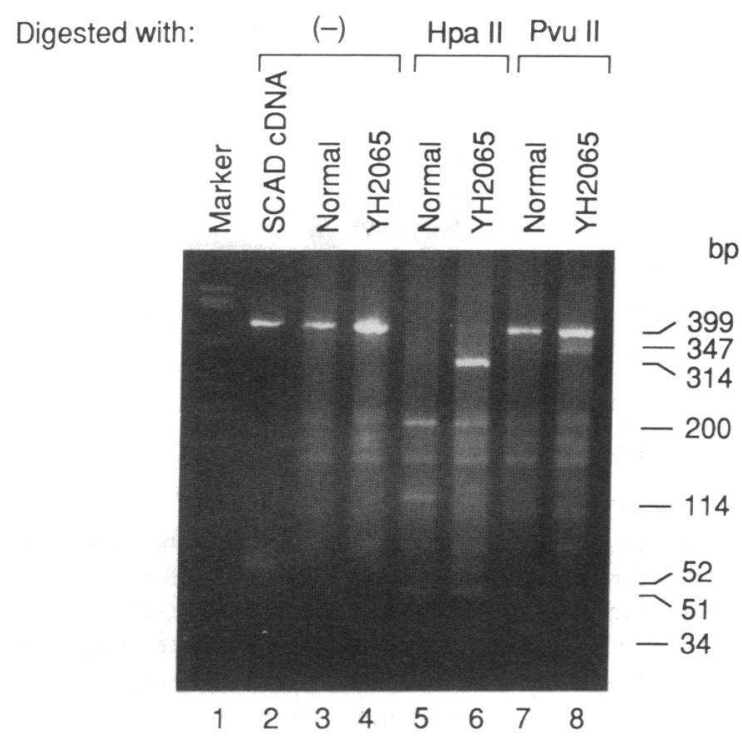

B

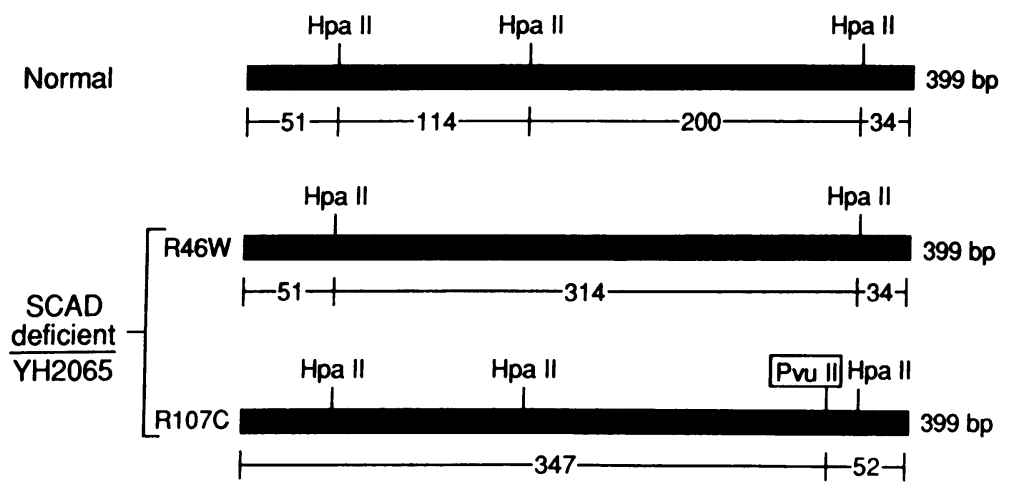

Figure 4. Restriction digestion of the PCR-amplified copies of the 5'-end fragment of pSCAD cDNA from normal and SCAD-deficient (YH2065) cell lines. $(A)$ Electrophoretic separation of the restriction fragments from the PCR-amplified copies on ethidium bromide-stained 4\% NuSieve GTG agarose gel. Hpa II (lanes 5 and 6) or Pvu II (lanes 7 and 8 ) was used for digestion. No restriction enzymes were used in lanes 2-4. The DNA samples were derived from the amplification of SCAD cDNA (HS-1) (lane 2), and cDNAs from normal (GM05756) (lanes 3, 5, and 7) and SCAD-deficient fibroblasts (YH2065) (lanes 4, 6 , and 8 ). The molecular weight markers shown in lane 1 were the Pst I-digested $\lambda$ DNA. The size of each digested fragment is indicated on the right side. In the lower part $(B)$, the location of the restriction sites and the size of the resulting fragments are schematically illustrated.
As shown in Fig. 5, the major band in the amplified product from human SCAD cDNA (HS-1) was 118 bp as expected. The major band of the PCR products from genomic DNA of YH2065 and normal cell lines was of the same size, indicating that the segment between nucleotide 252-369 is located in a single exon. The presence of $\mathrm{C} \rightarrow \mathrm{T}-319$ in the amplified copies from YH2065 was studied both by sequencing and by Pvu II digestion. For sequencing, the PCR-amplified copies were digested with Hinc II and Taq I, and then subcloned into pBluescript. Sequence analysis of the amplified fragments confirmed the presence of the $\mathrm{C} \rightarrow \mathrm{T}-319$ transition in two of the four clones obtained. The sequences of two other clones were normal, consistent with the presence of two distinct alleles in YH2065. When digested with Pvu II, the 118-bp fragment amplified from normal genomic DNA remained intact (Fig. 5, lane 5), whereas the 118-bp fragment amplified from YH2065 DNA yielded two faint short bands (66 and $52 \mathrm{bp}$ ) in addition to the undigested 118-bp fragment.

Southern blot analysis of genomic DNA. We also tested the direct effects of Pvu II and Hpa II digestion of genomic DNA from three normal cell lines, YH2065 and two other SCADdeficient cells. Among five cell lines other than YH2065, three patterns were observed with Pvu II digestion (Fig. 6). In three cell lines (two normal and one SCAD-deficient), three fragments $(8.7,4.3$, and $1.35 \mathrm{~kb})$ were detected. In one of the remaining two, a SCAD-deficient cell line, no $8.7-\mathrm{kb}$ band was observed, whereas in the other, a normal cell line, the 4.3-kb band was undetectable. Thus, the $4.3-$ and $8.7-\mathrm{kb}$ bands are allelic. These three patterns appear to be due to normal polymorphism, because we observed these three patterns also in genomic Southern blot analysis in nine normal human lymphoblastoid lines (data not shown). In YH2065, a 1.2-kb band was detected in addition to the three bands mentioned above (Fig. 6). This 1.2-kb band was not observed in any other cell lines including the five other fibroblast lines presented here and the nine normal lymphoblastoid lines. The presence of this unique 1.2-kb Pvu II fragment is consistent with the existence of the $\mathrm{C} \rightarrow \mathrm{T}$ transition in the variant SCAD genome that is responsible for the $\mathrm{C} \rightarrow \mathrm{T}-319$ transition.

Hpa II recognizes a specific sequence of only four bases (CCGG). Therefore, numerous Hpa II restriction sites were expected in genomic DNA. In fact, when the Hpa II-digested genomic DNA from three normal and two SCAD-deficient cell lines other than YH2065 was subjected to Southern blot analysis, multiple minor bands were detected and some variations of the pattern were observed. Only two bands, one at 10.0 $\mathrm{kb}$ and the other at $3.7 \mathrm{~kb}$, were commonly detected. The pattern in YH2065 was conspicuously different from those seen in the other five cell lines: the Hpa II-digested YH2065DNA contained only one very prominent band at $5.5 \mathrm{~kb}$ in 


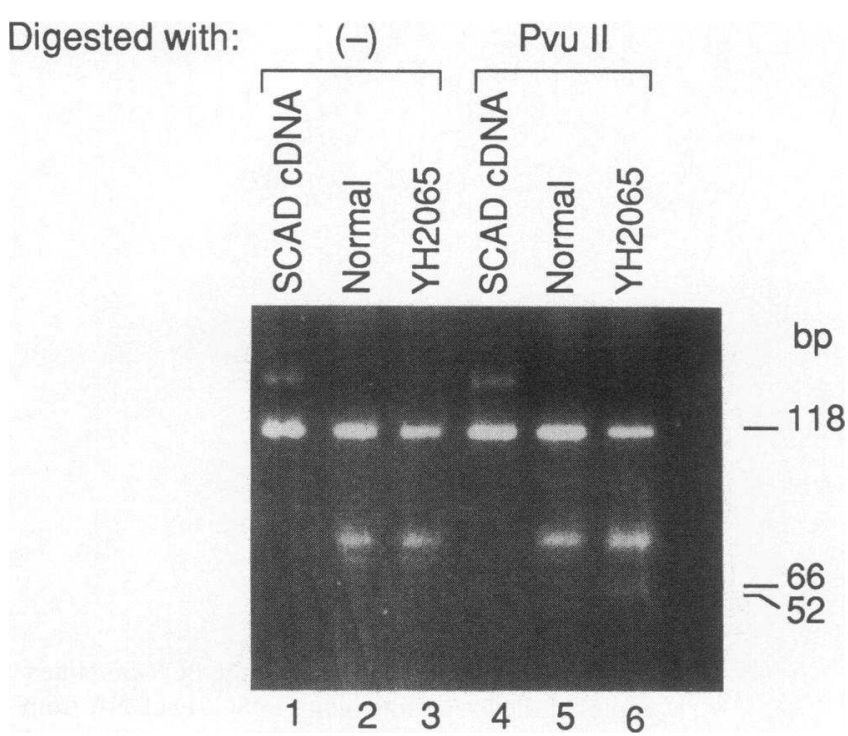

Figure 5. Pvu II-digestion of the PCR-amplified products from genomic DNA in normal and SCAD-deficient (YH2065) cell lines. The electrophoretic separation of the restriction fragments from the PCRamplified 5'-end copy of the genomic DNA using Pvu II is shown. Ethidium bromide-stained 4\% NuSieve GTG agarose gel was used. Lanes 1-3, PCR-amplified products without digestion and lanes 4-6, PCR-amplified products digested with Pvu II. The DNA samples were derived from cloned pSCAD cDNA (HS-1) (lanes $l$ and 4), and from genomic DNA in normal (GM05756) (lanes 2 and 5) and YH2065 fibroblasts (lanes 3 and 6).

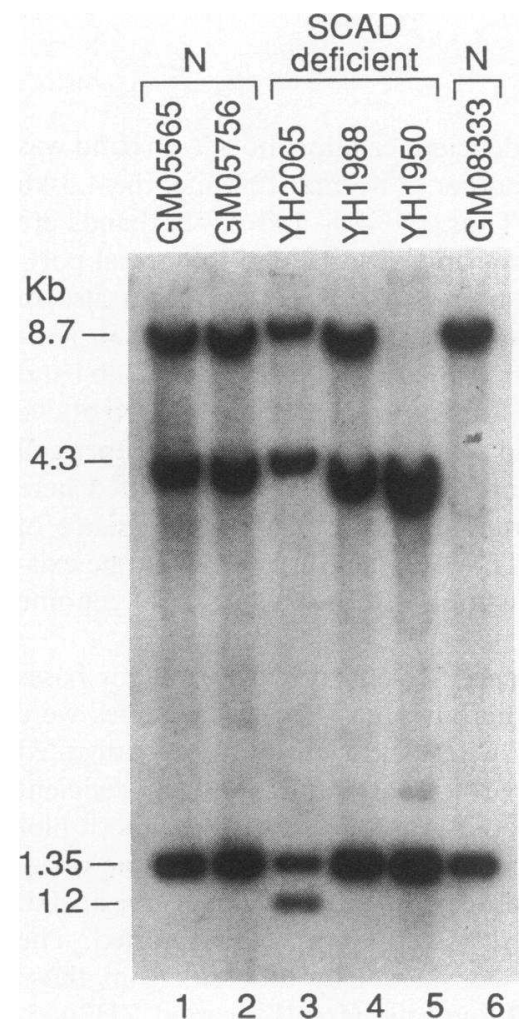

Figure 6. Southern blot analysis of genomic DNA. $10 \mu \mathrm{g}$ of genomic DNA isolated from fibroblasts were digested with Pvu II and subjected to Southern blot analysis. Genomic DNAs from three normal (lanes 1,2, and 6) and three SCAD-deficient cell lines (lanes 3-5) were analyzed. Cell lines are identified with the numbers at the top of each lane. Approximate sizes are given in kilobases on the left side.

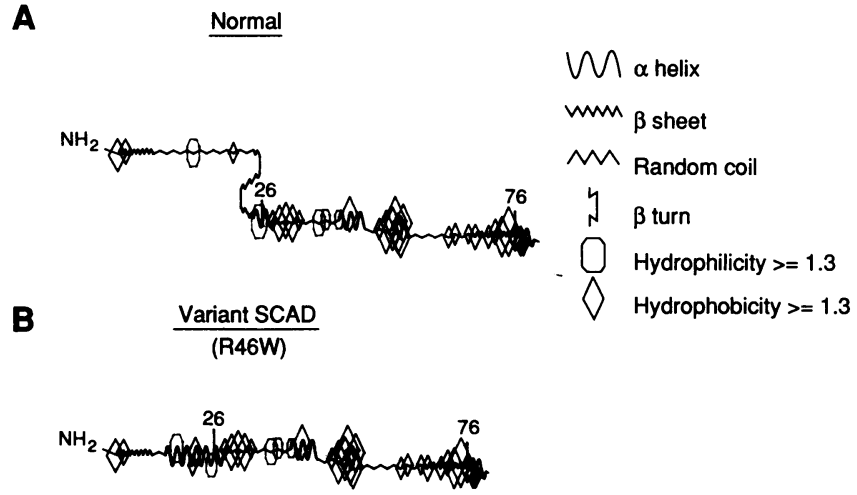

Figure 7. Computer-assisted prediction of the secondary structure of the $5^{\prime}$ region of normal and variant SCAD-R46W. The amino acid residue from the $\mathrm{NH}_{2}$-terminus and 75 th residue of the mature normal and variant SCAD-R46W were analyzed using the Peptidestructure and Plotstructure of the Genetic Computer Group nucleic acid and protein analysis program. The regions of random coil, $\beta$-sheet, $\beta$-turn, and $\alpha$-helix are illustrated in the figure. The hydrophobicity and hydrophilicity values are included in the secondary structure plot as diamonds and ovals, respectively. The $C \rightarrow T$ transition-136 causes substitution of Arginine-22 to tryptophan in the mature SCAD. Note that the $\beta$-turn at that position in normal SCAD is abolished in SCAD-R46W.

addition to a very strong $10-\mathrm{kb}$ band and a faint $3.7-\mathrm{kb}$ band (data not shown). However, the significance of this data is not clear at present.

Computer-assisted prediction of the secondary structure. Computer-assisted analysis of the region (residue 1-80) surrounding the Arg $\rightarrow$ Trp substitution at the 22 nd position of the mature SCAD (R46W) predicts drastic changes in the secondary structure of the $\mathrm{NH}_{2}$-terminal region. These changes include the loss of two $\beta$-turns and a random coil, with an increased tendency to form an $\alpha$-helical conformation (Fig. 7). No such drastic change was predicted in the secondary structure of the variant SCAD (R107C) due to the Arg $\rightarrow$ Cys substitution.

\section{Discussion}

Using PCR amplification of SCAD cDNAs, we demonstrated that $\mathrm{YH} 2065$ cells contain two variant SCAD alleles, one producing the SCAD mRNA with $\mathrm{C} \rightarrow \mathrm{T}$ transition-136 and the other producing the SCAD mRNA with $\mathrm{C} \rightarrow \mathrm{T}$ transition 319 . These mutations have been unambiguously confirmed through repeated sequencing of clones isolated from independent RNA isolations, reverse transcription experiments, and PCR amplification. Additionally, analysis of the PCR products and genomic DNA using two restriction enzymes, Hpa II and Pvu II, has shown that the natural restriction site for $\mathrm{Hpa}$ II was abolished as expected by $\mathrm{C} \rightarrow \mathrm{T}$ transition-136 and a new site for Pvu II was created by $\mathrm{C} \rightarrow \mathrm{T}$ transition-319. All the 5 '-fragment clones had either of the two $\mathrm{C} \rightarrow \mathrm{T}$ transitions, and were detected with equal frequency, indicating that this patient is a compound mutant for these two variant alleles.

In previous studies using immunoblot analysis and pulse/ chase experiments, we have shown that unlike the variant 
SCAD in two other SCAD-deficient patients, the variant SCAD-YH2065 in whole cells was extremely labile (4). However, in the previous biochemical experiments, we did not observe clear evidence for two distinct types of variant SCAD with differing stability. Therefore, it appears at present that both SCAD-R46W and -R107C are labile. It is likely that the mature variant SCAD with cysteine- 83 is unstable, since this cysteine is unpaired and may cause dimerization, leading to its instability. The substitution of Arginine-22 with tryptophan causes a drastic change in the secondary structure of the region, possibly leading to a change of the tertiary structure. It may be worthwhile to point out that Arginine-22 in the mature SCAD is an evolutionarily well-conserved residue. We recently reported cloning and sequences of several acyl-CoA dehydrogenases including human and rat $\operatorname{SCAD}(3,7)$, and rat MCAD (16), long chain acyl-CoA dehydrogenase, and IVD (7). Comparison of the sequences of these five acyl-CoA dehydrogenases plus human MCAD (17) revealed a high degree of sequence homology, with 57 invariant and 94 near-invariant residues among them. Arg- 22 is one of the near-invariant residues, conserved in five acyl-CoA dehydrogenases with the single exception of rat IVD (7).

It is possible that more accurate measurement of the rate of the loss of ${ }^{35} \mathrm{~S}$-labeled SCAD in the whole cell-labeling experiment, or the direct measurement of the stability of SCADR46W and -R107C, that are synthesized in vitro using the isolated variant SCAD cDNA, may reveal a difference in their stability. Still another possibility is that only one of the variant SCAD subunit is unstable, but that most of the variant SCAD-YH2065 tetramers become unstable due to the presence of one or more molecules of the unstable variant subunit participating in the tetramer.

At the nucleotide level, the sequences of the two Arg codons involved in the $\mathrm{C} \rightarrow \mathrm{T}$ transitions at positions 136 and 316 both participate in the formation of a tetranucleotide, GCCG, with both normal $\mathrm{C}$ residues contributing the second C. This tetranucleotide contains $\mathrm{CpG}$, the major site of methylation in human DNA. It is interesting to note in this regard that an unusually high frequency of restriction fragment length polymorphism has been observed at various loci in human DNA with the use of restriction enzymes whose recognition sequences contain a $\mathrm{CpG}$ dinucleotide. It has been speculated that $\mathrm{CpG}$ dinucleotides are probably mutation hot spots because the methylation of cytosine, followed by deamination of methylcytosine to thymidine, would result in a $\mathrm{C} \rightarrow \mathrm{T}$ transition (18). Point mutations due to $\mathrm{C} \rightarrow \mathrm{T}$ transition in the $\mathrm{CpG}$ dinucleotides have been identified in Factor VIII gene and ornithine transcarbamylase gene (19-21). Thus, the identifcation of the two point mutations described in this study are further examples of mutations caused by $\mathrm{C} \rightarrow \mathrm{T}$ transitions involving $\mathrm{CpG}$ dimers.

\section{Acknowledgments}

Synthesis and purification of oligonucleotides were carried out by Monica Talmor, Department of Pathology, Yale University School of Medicine. Cultured skin fibroblasts from three patients with SCAD deficiency were kindly provided by Drs. Carol Green (University of Colorado, Denver, CO), Vivian E. Shih (Massachusetts General Hospital, Boston, MA), and Susan Winter (Valley Children's Hospital,
Fresno, CA). We thank Mrs. Connie Woznick for preparing this manuscript.

This work was supported by a grant from the National Institutes of Health (DK-38154).

\section{References}

1. Amendt, B. A., C. Greene, L. Sweetman, J. Cloherty, V. Shih, A. Moon, L. Teel, and W. J. Rhead. 1987. Short-chain acyl-coenzyme A dehydrogenase deficiency. Clinical and biochemical studies in two patients. J. Clin. Invest. 79:1303-1309.

2. Coates, P. M., D. E. Hale, G. Finocchiaro, K. Tanaka, and S. C. Winter. 1988. Genetic deficiency of short-chain acyl-coenzyme A dehydrogenase in cultured fibroblasts from a patient with muscle carnitine deficiency and severe skeletal muscle weakness. J. Clin. Invest. 81:171-175.

3. Naito, E., H. Ozasa, Y. Ikeda, and K. Tanaka. 1989. Molecular cloning and nucleotide sequence of complementary DNAs encoding human short chain acyl-coenzyme A dehydrogenase and the study of the molecular basis of human short chain acyl-coenzyme A dehydrogenase deficiency. J. Clin. Invest. 83:1605-1613.

4. Naito, E., Y. Indo, and K. Tanaka. 1989. Short chain acyl-coenzyme A dehydrogenase (SCAD) deficiency: Immunochemical demonstration of molecular heterogeneity due to variant SCAD with differing stability. J. Clin. Invest. 84:1671-1674.

5. Ikeda, Y., K. Okamura-Ikeda, and K. Tanaka. 1985. Purification and characterization of short-chain, medium-chain, and longchain acyl-CoA dehydrogenases from rat liver mitochondria. J. Biol. Chem. 260:1311-1325.

6. Finocchiaro, G., M. Ito, and K. Tanaka. 1987. Purification and properties of short chain acyl-CoA, medium chain acyl-CoA, and isovaleryl-CoA dehydrogenases from human liver. J. Biol. Chem. 262:7982-7989.

7. Matsubara, Y., Y. Indo, E. Naito, H. Ozasa, R. Glassberg, J. Vockley, Y. Ikeda, J. Kraus, and K. Tanaka. 1989. Molecular cloning and nucleotide sequence of cDNAs encoding the precursors of rat long chain acyl-coenzyme $A$, short chain acyl-coenzyme $A$ and isovalerylcoenzyme A dehydrogenases: sequence homology of four enzymes of the Acyl-CoA Dehydrogenase Family. J. Biol. Chem. 264:1632116331.

8. Ikeda, Y., S. M. Keese, W. A. Fenton, and K. Tanaka. 1987. Biosynthesis of four rat liver mitochondrial acyl-CoA dehydrogenases: In vitro synthesis, import into mitochondria, and processing of their precursors in a cell-free system and in cultured cells. Arch. Biochem. Biophys. 252:662-674.

9. Saiki, R. K., D. H. Gelfand, S. Stoffel, S. J. Scharf, R. Higuchi, G. T. Horn, K. B. Mullis, and H. A. Erlich. 1988. Primer-directed enzymatic amplification of DNA with a thermostable DNA polymerase. Science (Wash. DC). 239:487-491.

10. Gibbs, R. A., P.-N. Nguyen, L. J. McBride, S. M. Koepf, and C. T. Caskey. 1989. Identification of mutations leading to the LeschNyhan syndrome by automated direct DNA sequencing of in vitro amplified cDNA. Proc. Natl. Acad. Sci. USA. 86:1919-1923.

11. McBride, L. J., C. McCollum, S. Davidson, J. W. S. Efcavitch, A. Andrus, and S. J. Lombardi. 1988. A new, reliable cartridge for the rapid purification of synthetic DNA. Biotechniques. 6:362-367.

12. Sanger, F., S. Nicklen, and A. R. Coulson. 1977. DNA sequencing with chain-terminating inhibitors. Proc. Natl. Acad. Sci. USA. 74:5463-5467.

13. Chou, P. Y., and G. D. Fasman. 1978. Empirical predictions of protein conformation. Annu. Rev. Biochem. 47:251-276.

14. Wolf, H., S. Modrow, M. Motz, B. Jameson, B. Hermann, and B. Fotsch. 1988. An integrated family of amino acid sequence analysis programs. Comput. Appl. Biosci. 4:187-191. 
15. Hopp, T. P., and K. R. Woods. 1981. Prediction of protein antigenic determinants from amino acid molecular weights. Proc. Natl. Acad. Sci. USA. 78:3824-3828.

16. Matsubara, Y., J. P. Kraus, H. Ozasa, R. Glassberg, G. Finocchairo, Y. Ikeda, J. Mole, L. E. Rosenberg, and K. Tanaka. 1987. Molecular cloning and nucleotide sequence of cDNA encoding the entire precursor of rat liver medium chain acyl coenzyme A dehydrogenase. J. Biol. Chem. 262:10104-10108.

17. Kelly, D. P., J. J. Kim, J. J. Billadello, B. E. Hainline, T. W. Chu, and A. W. Strauss. 1987. Nucleotide sequence of medium-chain acyl-CoA dehydrogenase mRNA and its expression in enzyme-deficient human tissue. Proc. Natl. Acad. Sci. USA. 84:4068-4072.

18. Barker, D., M. Schafer, and R. White. 1984. Restriction sites containing $\mathrm{CpG}$ show a higher frequency of polymorphism in human DNA. Cell. 36:131-138.

19. Gitschier, J., W. I. Wood, M. A. Shuman, and R. M. Lawn. 1986. Identification of a missense mutation in the factor VIII gene of a mild hemophiliac. Science (Wash. DC). 232:1415-1416.

20. Maddalena, A., J. E. Spence, W. E. O'Brian, and R. L. Nussbaum. 1988. Characterization of point mutations in the same arginine codon in three unrelated patients with ornithine transcarbamylase deficiency. J. Clin. Invest. 82:1353-1358.

21. Hata, A., C. Setoyama, K. Shimada, E. Takeda, Y. K. I. Akaboshi, and I. Matsuda. 1989. Ornithine transcarbamylase deficiency resulting from a C-to-T substitution in exon 5 of the ornithine transcarbamylase gene. Am. J. Hum. Genet. 45:123-127. 